\title{
ТЕОРЕТИЧЕСКАЯ МЕХАНИКА
}

Научная статья

УДК 532.782, 519.642

DOI: $10.18101 / 2304-5728-2020-3-32-41$

\section{ИНТЕГРАЛЬНОЕ УРАВНЕНИЕ ФРЕДГОЛЬМА ВТОРОГО РОДА В СТАТИСТИЧЕСКОЙ ФИЗИКЕ ЖИДКОСТЕЙ}

\section{(C) Аграфонов Юрий Васильевич}

доктор физико-математических наук, профессор, профессор кафедры радиофизики и радиоэлектроники, Иркутский государственный университет Россия, 664003, г. Иркутск, ул. К. Маркса, 1 agrafonov@physdep.isu.ru

\section{(C) Петрушин Иван Сергеевич}

кандидат технических наук, доцент, доцент кафедры радиофизики и радиоэлектроники, Иркутский государственный университет Россия, 664003, г. Иркутск, ул. К. Маркса, 1 ivan.kiel@gmail.com

\section{(C) Орлов Сергей Сергеевич}

кандидат физико-математических наук, доцент, доцент кафедры математического анализа и дифференциальных уравнений, Иркутский государственный университет Россия, 664003, г. Иркутск, ул. К. Маркса, 1 orlov_sergey@inbox.ru

\section{(с) Цыдыпов Шулун Балдоржиевич} доктор технических наук, доцент, доцент кафедры общей и теоретической физики, Бурятский государственный университет имени Доржи Банзарова Россия, 670000, г. Улан-Удэ, ул. Смолина, 24а shulun52@gmail.com

\section{(C) Герман Евгений Иванович}

старший преподаватель кафедры общей и теоретической физики, Бурятский государственный университет имени Доржи Банзарова Россия, 670000, г. Улан-Удэ, ул. Смолина, 24а net-admin@list.ru

Благодарности. Работа выполнена при частичной финансовой поддержке РФФИ, грант №18-02-00523а. Авторы выражают благодарность Д. С. Сандитову и Б. Б. Дамдинову за обсуждение и полезные комментарии к статье. Авторы признательны А. А. Гаврилюку за интерес, проявленный к работе. 
Ю. В. Аграфонов, И. С. Петрушин, С. С. Орлов, Ш. Б. Цыдыпов, Е. И. Герман. Интегральное уравнение Фредгольма второго рода в статистической физике ...

Аннотация. Проведен анализ применимости алгоритмов решения различных приближений для нелинейных уравнений статистической физики жидкостей к решению линейного интегрального уравнения Фредгольма второго рода, предложенного ранее для описания поверхностных явлений в жидкостях. Рассмотрена молекулярная система твердых сфер, граничащих с твердой поверхностью. В приближении Перкус - Йевика для ядра и правой части, решение ищется в классе кусочно-непрерывных функций. Сформулирован метод аналитического вычисления на каждом интервале в области определения функции. Для других приближений ядро уравнения и правая часть вычисляются численно. Решение уравнения Фредгольма также должно решаться численно. Для его решения предложен алгоритм Лабика - Малиевского, являющийся эталоном точности в современной физике жидкостей. Предложено применять данный алгоритм для вычисления двухчастичной функции распределения метастабильных состояний в теории хаотического фазового перехода первого рода переохлажденная жидкость - идеальное стекло, что позволит описывать поверхностные явления в аморфных пленках.

Ключевые слова: переохлажденная жидкость; идеальное стекло; частичные функции распределения; реплики; хаотический фазовый переход первого рода; уравнение Фредгольма второго рода.

\section{Для цитирования}

Аграфонов Ю. В., Петрушин И. С., Орлов С. С., Цыдыпов Ш. Б., Герман Е. И. Интегральное уравнение Фредгольма второго рода в статистической физике жидкостей // Вестник Бурятского государственного университета. Математика, информатика. 2020. № 3. С. 32-41.

Введение
В статистической физике жидкостей для описания структурных характеристик и термодинамических величин основную роль играют одночастичная $G_{1}\left(\vec{r}_{1}\right)$ и двухчастичная $G_{12}\left(\vec{r}_{1}, \vec{r}_{2}\right)$ функции распределения. Обе эти функции находятся совместным решением системы нелинейных интегральных уравнений Орнштейна - Цернике (ОЦ) [1-4]. Ядра уравнений представлены бесконечными функциональными рядами от этих же функций распределения. Аппроксимация рядов простыми аналитическими выражениями приводит к приближенным интегральным уравнениям. Однако методическая погрешность данных аппроксимаций неизвестна. Как правило, уравнения решаются численно, а полученное решение сравнивается с данными численного эксперимента, являющегося эталоном точности.

Для пространственно-однородных жидкостей, для которых поверхностные эффекты не учитываются, двухчастичная функция распределения зависит только от одной переменной $r_{12}=\left|\vec{r}_{1}-\vec{r}_{2}\right|$. Система двух уравнений ОЦ сворачивается к одному уравнению для двухчастичной функции распределения $G_{12}^{(0)}\left(r_{12}\right)$, что и обеспечивает сравнительно простые численные вычисления.

Для описания структуры жидкости, граничащей с твердой поверхно- 
стью, приходится решать исходную систему уравнений. В силу аксиальной симметрии, функции распределения есть $G_{1}\left(z_{1}\right), G_{12}\left(r_{12}, z_{1}, z_{2}\right)$, где $z_{1}, z_{2}$ - удаление первой и второй частицы от поверхности. Решение системы нелинейных уравнений для функций с таким числом переменных является сложной задачей. Эту сложность в большинстве случаев обходят c помощью синглетного приближения [5-6], заменяя функцию $G_{12}\left(r_{12}, z_{1}, z_{2}\right)$ ее граничным значением $G_{12}^{(0)}\left(r_{12}\right)$. В результате получается нелинейное интегральное уравнение для функции одной переменной $G_{1}\left(z_{1}\right)$, ядро которого зависит от $G_{12}^{(0)}\left(r_{12}\right)$. Решение данного уравнения возможно сравнительно просто получить численно. Тем не менее погрешность синглетного приближения остается неизвестной и полученное решение вновь приходится сравнивать с численным экспериментом. Для жидкостей с разными плотностями при различных температурах, обе методические погрешности могут либо компенсировать друг друга, либо нет.

Таким образом, описание структуры жидкостей в контакте с твердой поверхностью остается актуальной задачей.

В наших работах [7-8] было предложено переопределить неприводимые диаграммы в уравнении для одночастичной функции распределения таким образом, чтобы они компенсировали все нелинейности и свели синглетное приближению к линейному интегральному уравнению. В результате получается линейное интегральное уравнение Фредгольма второго рода. Проведен анализ литературы по аналитическому и численному решению уравнений Фредгольма второго рода с целью реализации алгоритма, требующего наименьших вычислительных ресурсов.

\section{1 Линейное интегральное уравнение физики жидкостей на границе с твердой поверхностью}

Для вычисления локальной микроструктуры граничных слоев жидкостей и термодинамических параметров необходимо знать одночастичную функцию распределения $G_{1}\left(\vec{r}_{1}\right)=\exp \left(-\Phi_{1}\left(\vec{r}_{1}\right) / k T+\omega_{1}\left(\vec{r}_{1}\right)\right)$, которая определяет вероятность нахождения частицы во внешнем поле $\Phi_{1}\left(\vec{r}_{1}\right)$ вблизи точки с координатами $\vec{r}_{1}$. Одночастичный термический потенциал $\omega_{1}\left(\vec{r}_{1}\right)$ учитывает взаимодействие частицы с окружающей средой. Линейное интегральное уравнение для одночастичной функции распределения $\exp \left(\omega_{1}\left(z_{1}\right)\right)$ для жидкости, граничащей с твердой поверхностью $\left(\Phi_{1}\left(\vec{r}_{1}\right)=0\right)$ было получено в [7-8] 
Ю. В. Аграфонов, И. С. Петрушин, С. С. Орлов, Ш. Б. Цыдыпов, Е. И. Герман. Интегральное уравнение Фредгольма второго рода в статистической физике ...

$$
\begin{aligned}
& \left(e^{\omega_{1}\left(z_{1}\right)}-1\right)-2 \pi n \int_{0}^{\infty} d z_{2}\left(e^{\omega_{1}\left(z_{2}\right)}-1\right) \int_{\left|z_{12}\right|}^{\infty} r_{12} d r_{12} C_{12}\left(r_{12}\right)= \\
& =-2 \pi n \int_{z_{1}}^{\infty} d z_{12} \int_{z_{12}}^{\infty} r_{12} d r_{12} C_{12}\left(r_{12}\right) .
\end{aligned}
$$

Здесь учтено, что частицы жидкости взаимодействуют с поверхностью посредством потенциала твердых сфер. Прямая корреляционная функция $C_{12}\left(r_{12}\right)$ должна быть предварительно вычислена. Уравнение (1) есть интегральное уравнение Фредгольма второго рода. Ядро уравнения и правая часть предварительно вычисляются из соответствующего интегрального уравнения для двухчастичной функции распределения макроскопической жидкости [1-4]. В том случае, когда ядро и правая часть уравнения вычисляются аналитически, данное уравнение также может быть решено аналитически. В частности, для замыкания Перкус - Йевика [9] для молекулярной системы твердых сфер, внутренние интегралы уравнения (1) можно представить в виде:

$$
K(|z|)=-\theta(1-|z|) \Psi(|z|), \Psi(|z|)=\int_{|z|}^{1} r_{12} d r_{12}\left(\alpha+\beta r_{12}+\gamma r_{12}{ }^{3}\right),
$$

где

$$
\alpha=(2 \eta+1)^{2} /(\eta-1)^{4},
$$

$$
\beta=-3 \eta(2+\eta)^{2} / 2(\eta-1)^{4}
$$

$\gamma=\eta(2 \eta+1)^{2} / 2(\eta-1)^{4}$.

В результате уравнение принимает вид:

$$
\begin{gathered}
f\left(z_{1}\right)=-12 \eta \int_{0}^{1+z_{1}} d z_{2} f\left(z_{2}\right) \Psi\left(\left|z_{1}-z_{2}\right|\right)+12 \eta \int_{z_{1}}^{1} d z_{2} \Psi\left(z_{2}\right), \quad z_{1} \leq 1, \\
f\left(z_{1}\right)=-12 \eta \int_{z_{1}-1}^{z_{1}+1} d z_{2} f\left(z_{2}\right) \Psi\left(\left|z_{1}-z_{2}\right|\right), \quad z_{1} \geq 1,
\end{gathered}
$$

где мы обозначили: $\eta=\pi n / 6, f\left(z_{1}\right)=\exp \left(\omega_{1}\left(z_{1}\right)\right)-1$. Из (3) вытекает, что для вычисления функции на интервале $0 \leq z_{1} \leq 1$ необходимо знать, как она изменяется на интервале $1 \leq z_{1} \leq 2$. В свою очередь, как следует из (4), для этого необходимо знать изменение функции на интервале $2 \leq z_{1} \leq 3$ и т. д. Таким образом получается бесконечная последовательность функций $f_{n}\left(z_{1}\right), n \leq z_{1} \leq n+1, \quad(n=0,1,2,3, \ldots$.$) . Поэтому решение уравнений$ (3)-(4) естественно искать в классе кусочно-непрерывных функций

$$
f\left(z_{1}\right)=\sum_{n=0}^{\infty} f_{n}\left(z_{1}\right) \theta\left(z_{1}-n\right) \theta\left(n+1-z_{1}\right) .
$$

Для вычисления этой последовательности необходимо привлекать дополнительные соображения о поведении функции $f_{0}\left(z_{1}\right)$ на интервале 
$0 \leq z_{1} \leq 1$. Для вычисления $f_{0}\left(z_{1}\right)$ мы применяем такой же подход, как и для решения нелинейного уравнения ПЙ. Совершаем преобразование Лапласа $\int_{0}^{\infty} d z_{1} \theta\left(z_{1}-n\right) \theta\left(n+1-z_{1}\right) f_{n}\left(z_{1}\right) \exp \left(-\lambda z_{1}\right)$. Все преобразования Лапласа от старших функций выражаются через преобразование Лапласа от функции $f_{0}\left(z_{1}\right)$ в виде отношения двух полиномов разных степеней от переменной $\exp (-\lambda)$. Условие деления полиномов без остатка (что необходимо для того, чтобы все функции были аналитическими) позволяет записать функцию $f_{0}\left(z_{1}\right)$ в виде конечного ряда по степеням $z_{1}$. Подстановка полученного решения в (3)-(4) приводит к уравнениям Вольтерра для старших функций, которые также могут быть решены аналитически. Мы не приводим здесь довольно сложные аналитические выражения.

Для численного решения уравнения (1) с различными замыканиями и параметрами межмолекулярного взаимодействия необходимо предварительно вычислить на основе решения уравнений физики жидкостей.

\section{2 Интегральные уравнения физики жидкостей}

Микроструктура и термодинамические параметры макроскопических жидкостей определяются двухчастичной функцией распределения $G_{12}=\exp \left(-\Phi_{12} / k T+\omega_{12}\right)$, которая определяет корреляцию в положении двух частиц вблизи точек с координатами $\vec{r}_{1}, \vec{r}_{2}$, учитывая их прямое взаимодействие посредством парного потенциала $\Phi_{12}$ и коллективными эффектами, обусловленными взаимодействием с окружающей средой $\omega_{12}$. Функция $G_{12}$ находится из уравнения Орнштейна - Цернике (ОЦ) [1-4]

$$
h_{12}=C_{12}+n \int C_{13} h_{23} d(3) \text {. }
$$

Элемент объема интегрирования равен $d(i)=d \vec{r}_{i}, n-$ плотность, парная корреляционная функция $h_{i j}=G_{i j}-1$. Прямая корреляционная функция $C_{i j}$ записывается в виде $C_{i j}=h_{i j}-\omega_{\mathrm{ij}}+M_{i j}$, где $M_{i j}$ есть бесконечный функциональный ряд от парной функции распределения. Как правило в рядах учитывают только приводимые диаграммы, которые можно просуммировать. В результате получается соотношение между парной и прямой функциями распределения (замыкание), что сводит уравнение ОЦ к тому или иному нелинейному интегральному уравнению, для решения которых необходимо прибегать численным методам. Наиболее известными из них являются Перкус - Йевика, гиперцепное, Мартынова - Саркисова и Роджерс - Янга [1-4]. Методическая погрешность данных уравнений до сих пор остается неизвестной.

Прямая корреляционная функция, входящая в ядро и правую часть уравнения (5), является решением соответствующего нелинейного инте- 
Ю. В. Аграфонов, И. С. Петрушин, С. С. Орлов, Ш. Б. Цыдыпов, Е. И. Герман. Интегральное уравнение Фредгольма второго рода в статистической физике ...

грального уравнения (6). Отметим, что параметрами, определяющими решение уравнения (6), являются потенциал межмолекулярного взаимодействия, температура и плотность. Так как уравнение Орнштейна (6) есть свертка, то оно может быть решено с помощью Фурьепреобразования. Вычисление Фурье-компонент проводится посредством их разложения по базисным функциям с последующим применением метода прямых итераций. Итерационная процедура продолжается до достижения требуемой точности. Сходимость итерационной процедуры зависит от удачного выбора начального приближения. Для молекулярных систем со сферически симметричными потенциалами взаимодействия Лабик и Малиевский $[10 ; 11]$ предложили быстро сходящийся метод решения уравнения ОЦ. На данный момент этот алгоритм является наилучшим.

Алгоритм Лабика - Малиевского численного решения уравнения (6) с различными замыканиями позволяет вычислить прямую корреляционную функцию $C_{12}\left(r_{12}\right)$ для молекулярных систем со сферическими потенциалами межмолекулярного взаимодействия в широком диапазоне плотностей и температур. Данное решение позволяет описать фазовые переходы газ - жидкость и жидкость - кристалл в термодинамически равновесных системах.

Подстановка $C_{12}\left(r_{12}\right)$ в уравнение (1) позволяет получить решение для функции распределения $G_{1}\left(z_{1}\right)=\exp \left(\omega_{1}\left(z_{1}\right)\right)$, описывающей изменение локальной микроструктуры жидкости вблизи твердой поверхности. Сопоставление полученного результата с численным решением синглетного уравнения [5] позволит сделать вывод о преимуществах или недостатках нашего подхода для описания поверхностных явлений в термодинамически равновесных системах.

\section{3 Термодинамически неравновесные системы}

Для термодинамически равновесных систем справедлива эргодическая гипотеза, в основе которой лежит предположение, что усреднение макроскопических величин по времени эквивалентно их вычислению по статистическому ансамблю. Однако для метастабильных состояний (аморфные тела, стекла) эргодическая гипотеза не справедлива, поскольку нет упорядоченного расположения частиц в пространстве: переход в метастабильное состояние есть кинетический процесс, а не фазовый переход. При описании процесса аморфизации расплава необходимо учитывать как скорость теплоотвода, так и время структурной перестройки, обусловленное вязкостью жидкости. Однако для молекулярных систем, в которых отсутствует вязкость, была предложена модель хаотического фазового перехода (ХФП) первого рода [12-14]. Такой переход из начального (равновесного) в конечное (метастабильное) состояние позволяет не учитывать промежуточных кинетических процессов. Были сформулированы уравнения для прямой корреляционной функции $C_{12}\left(r_{12}\right)$, позволяющие отличить переохлажденную жидкость от стекла. Современное состояние в 
этой области исследований приведено в наших обзорах [7-8]. Основная идея теории ХФП заключается в модификации метода частичных функций распределения. Макроскопическое тело рассматривается в виде тождественных подсистем (копий или реплик). В каждой из реплик межмолекулярное взаимодействие осуществляется одинаково, тем самым сохраняется микроскопическая однородность во всем объеме макроскопического тела. Однако теперь между репликами существует специальным образом подобранное взаимодействие, которое уменьшает характерное расстояние между частицами по сравнению с тем, что было в жидкости. Тем самым формулируются количественные характеристики, позволяющие отличить идеальное стекло от переохлажденной жидкости. Соответственно, записываются модифицированные уравнения ОЦ. Самый простой случай, когда имеется две реплики. Уравнения для парных функций распределения $g_{11}(x)$ и $g_{12}(x)$ записываются в виде [3]

$$
\begin{aligned}
& h_{11}(x)=c_{11}(x)+\rho^{*}\left[c_{11} \otimes h_{11}(x)+c_{12} \otimes h_{12}(x)\right], \\
& h_{12}(x)=c_{12}(x)+\rho^{*}\left[c_{11} \otimes h_{12}(x)+c_{12} \otimes h_{11}(x)\right],
\end{aligned}
$$

где символ $\otimes$ означает свертки двух функций, $\rho^{*}-$ плотность тождественных реплик, $g_{11}(x) \equiv g_{22}(x)$ и $g_{12}(x) \equiv g_{21}(x)$. Структура уравнений (7), (8) такая же, как и уравнения ОЦ для термодинамически равновесной жидкости, поэтому их можно решать с помощью алгоритма Лабика Малиевского. Отметим, что уравнения (7)-(8) описывают метастабильное состояние (стекло) макроскопической системы, в которой не учитываются поверхностные эффекты. Подстановка решения уравнений (7)-(8) в уравнение (1) позволит учесть поверхностные эффекты в метастабильной макроскопической системе (аморфные пленки).

\section{Заключение}

В настоящее время интегральные уравнения физики поверхностных явлений в жидкостях являются приближенными. Оценка погрешности, как правило, находится сравнением с данными численного эксперимента, являющегося в настоящий момент эталоном точности. Разрабатываемый нами подход к статистической физике поверхностных явлений основан на предположении, что учет неприводимых диаграмм в бесконечных функциональных рядах для прямых корреляционных функций можно проводить так, чтобы осуществлялась компенсация всех нелинейностей.

В результате получается стандартное уравнение Фредгольма второго рода. В том случае, когда ядро и правая часть заданы аналитически, уравнение также решается аналитически. В общем случае решение находится численно. Тем не менее вычислительные затраты несопоставимо меньше, чем при решении нелинейных уравнений. Данный факт является несомненным достоинством. В то же время методическую погрешность уравнения все-таки придется оценивать с данными численного эксперимента. Однако такое сопоставление позволит сказать у какого уравнения по- 
Ю. В. Аграфонов, И. С. Петрушин, С. С. Орлов, Ш. Б. Цыдыпов, Е. И. Герман. Интегральное уравнение Фредгольма второго рода в статистической физике ...

грешность меньше. Другим достоинством линейного уравнения является возможность обобщить его для описания тонких аморфных пленок, имеющих важное значение в научных исследованиях и технических разработках.

\section{Литература}

1. Martinov G. A. Fundamental Theory of Liquids; Method of Distribution Functions. Bristol, 1992. 470 p.

2. Vompe A. G., Martynov G. A. The Self-Consistent Statistical Theory of Condensation // The Journal of Chemical Physics. 1997. Vol. 106, № 14. P. 6095-6101. DOI: https://doi.org/10.1063/1.473272.

3. Parisi G., Zamponi F. The Ideal Glass Transition of Hard Spheres // The Journal of Chemical Physics. 2005. Vol. 123, № 14. P. 144-501. DOI: https://doi.org/10.1063/1.2041507.

4. Rogers F. J., Young D. A. New, Thermodynamically Consistent, Integral Equation for Simple Fluids // Physical Review A. 1984. Vol. 30, № 2. P. 999. DOI: https://doi.org/10.1103/PhysRevA.30.999.

5. Tikhonov D. A., Kiselyov O. E., Martynov G. A., Sarkisov G. N. Singlet Integral Equation in the Statistical Theory of Surface Phenomena in Liquids // J. of Mol. Liquids. 1999. Vol. 82. P. 3-17. DOI: https://doi.org/10.1016/S01677322(99)00037-9.

6. Agrafonov Yu., Petrushin I. Two-Particle Distribution Function of a Non-Ideal Molecular System near a Hard Surface // Physics Procedia. 2015. Vol. 71. P. 364-368. DOI: https://doi.org/10.1016/j.phpro.2015.08.353.

7. Agrafonov Yu. V., Petrushin I. S. Random First Order Transition from a Supercooled Liquid to an Ideal Glass // Condensed Matter and Interphases. 2020. Vol. 22, № 2. P. 291-302. DOI: https://doi.org/10.17308/kcmf.2020.22/2959.

8. Agrafonov Y. V., Petrushin I. S. Using Molecular Distribution Functions to Calculate the Structural Properties of Amorphous Solids // Bull. Russ. Acad. Sci. Phys. 2020. Vol. 8. P. 783-787. DOI: https://doi.org/10.3103/S1062873820070035.

9. Wertheim M. S. Exact solution of the Percus - Yevick Integral Equation for Hard Spheres // Phys. Rev. Letters. 1963. V. 10, № 8. P. 321-323. DOI: https://doi.org/10.1103/PhysRevLett.10.321.

10. Labik S., Malijevsky A., Vonka P. A rapidly convergent method of solving the OZ equation // Molecular Physics. 1985. Vol. 56, № 3. P. 709-715. DOI: https://doi.org/10.1080/00268978500102651.

11. Malijevsky A., Labik S. The Bridge Function for Hard Spheres // Molecular $\begin{array}{lllllll}\text { Physics. } 1987 . \quad \text { Vol. 60, № 3, P. 663-669. DOI: } & \end{array}$ https://doi.org/10.1080/00268978700100441.

12. Franz S., Mezard M., Parisi G., Peliti L. The Response of Glassy Systems to Random Perturbations: A Bridge between Equilibrium and Off-Equilibrium // Journal of Statistical Physics. 1999. Vol. 97, № 3-4. Pp. 459-488. DOI: https://doi.org/10.1023/A:1004602906332.

13. Mezard M., Parisi G. Thermodynamics of Glasses: a First Principles Computation // J. of Phys.: Condens. Matter. 1999. Vol. 11. P. A157-A165. DOI: https://doi.org/10.1088/0953-8984/11/10A/011.

14. Robles M., López de Haro M., Santos A., Bravo Yuste S. Is There a Glass Transition for Dense Hard-Sphere Systems? // The Journal of Chemical Physics. 1998. Vol. 108, № 3. P. 1290-1291. DOI: https://doi.org/10.1063/1.475499. 


\section{FREDHOLM INTEGRAL EQUATION OF SECOND KIND IN STATISTICAL PHYSICS OF FLUIDS}

Yury V. Agrafonov

Dr. Sci. (Phys. and Math.), Prof.,

Prof. of Radio Physics and Electronics Department, Irkutsk State University,

1 Karla Marksa St., Irkutsk 664003, Russia

agrafonov@physdep.isu.ru

Ivan S. Petrushin

Cand. Sci. (Engineering), A/Prof.,

A/Prof. of Radio Physics and Electronics Department,

Irkutsk State University

1 Karla Marksa St., Irkutsk 664003, Russia

ivan.kiel@gmail.com

Sergey S. Orlov

Dr. Sci. (Phys. and Math.), A/Prof.,

A/Prof. of Mathematical Analysis and Differential Equations Department,

Irkutsk State University

1 Karla Marksa St., Irkutsk 664003, Russia

orlov_sergey@inbox.ru

Shulun B. Tsydypov

Dr. Sci. (Engineering), A/Prof.,

A/Prof. of General and Theoretical Physics Department

Dorzhi Banzarov Buryat State University

24a Smolina St., Ulan-Ude 670000, Russia

shulun52@gmail.com

\section{Evgeny I. German}

Senior Lecturer of General and Theoretical Physics Department,

Dorzhi Banzarov Buryat State University

24a Smolina St., Ulan-Ude 670000, Russia

net-admin@list.ru

Abstract. The article analyzes the applicability of algorithms for solving various approximations for nonlinear equations of statistical physics of fluids to the solution of the linear integral Fredholm equation of second kind, which has been proposed earlier for description of surface phenomena in liquids. We have considered a molecular system of solid spheres bordering a solid surface. In the Percus-Yevick approximation for the kernel and right-hand side, the solution is sought in the class of piecewise continuous functions. We formulate the method of analytical calculation for each interval in the domain of function. For other approximations, the core of the equation and the right-hand side are calculated numerically. Fredholm equation should be also solved numerically. To solve it, we propose the Labik-Malijevsky algorithm, which is a standard of precision in modern physics of fluids. It is proposed to use this algorithm to calculate the two-particle function of meta-stable states distribution in the theory of the first kind chaotic phase transition of supercooled liquid - ideal glass, which will allow describing surface phenomena in amorphous films.

Keywords: supercooled liquid; ideal glass; partial distribution functions; replicas; chaotic first-kind phase transition; Fredholm equation of second kind. 
Ю. В. Аграфонов, И. С. Петрушин, С. С. Орлов, Ш. Б. Цыдыпов, Е. И. Герман. Интегральное уравнение Фредгольма второго рода в статистической физике ...

\section{References}

1. Martinov G. A. Fundamental Theory of Liquids; Method of Distribution Functions. Bristol, 1992, 470 p.

2. Vompe A. G., Martynov G. A. The Self-Consistent Statistical Theory of Condensation. The Journal of Chemical Physics. 1997. Vol. 106, no. 14. Pp. 6095-6101. DOI: https://doi.org/10.1063/1.473272.

3. Parisi G., Zamponi F. The Ideal Glass Transition of Hard Spheres. The Journal of Chemical Physics. 2005. Vol. 123, no. 14. Pp. 144-501. DOI: https://doi.org/10.1063/1.2041507.

4. Rogers F. J., Young D. A. New, Thermodynamically Consistent, Integral Equation for Simple Fluids. Physical Review A. 1984. Vol. 30, no. 2. P. 999. DOI: https://doi.org/10.1103/PhysRevA.30.999.

5. Tikhonov D. A., Kiselyov O. E., Martynov G. A., Sarkisov G. N. Singlet Integral Equation in the Statistical Theory of Surface Phenomena in Liquids. J. of Mol. Liquids. 1999. Vol. 82. Pp. 3-17. DOI: https://doi.org/10.1016/S0167-7322(99)00037-9.

6. Agrafonov Yu., Petrushin I. Two-Particle Distribution Function of a Non-Ideal Molecular System near a Hard Surface. Physics Procedia. 2015. Vol. 71. Pp. 364-368. DOI: https://doi.org/10.1016/j.phpro.2015.08.353.

7. Agrafonov Yu. V., Petrushin I. S. Random First Order Transition from a Supercooled Liquid to an Ideal Glass. Condensed Matter and Interphases. 2020. Vol. 22. No. 2. Pp. 291-302. DOI: https://doi.org/10.17308/kcmf.2020.22/2959.

8. Agrafonov Y. V., Petrushin I. S. Using Molecular Distribution Functions to Calculate the Structural Properties of Amorphous Solids. Bull. Russ. Acad. Sci. Phys. 2020. Vol. 8. Pp. 783-787. DOI: https://doi.org/10.3103/S1062873820070035.

9. Wertheim M. S. Exact solution of the Percus-Yevick Integral Equation for Hard Spheres. Phys. Rev. Letters. 1963. Vol. 10, no. 8. Pp. 321-323. DOI: https://doi.org/10.1103/PhysRevLett.10.321.

10. Labik S., Malijevsky A., Vonka P. A Rapidly Convergent Method of Solving the OZ Equation. Molecular Physics. 1985. Vol. 56, no. 3. Pp. 709-715. DOI: https://doi.org/10.1080/00268978500102651.

11. Malijevsky A., Labik S. The Bridge Function for Hard Spheres. Molecular Physics. 1987. Vol. 60, no. 3. Pp. 663-669. DOI: https://doi.org/10.1080/00268978700100441.

12. Franz S., Mezard M., Parisi G., Peliti L. The Response of Glassy Systems to Random Perturbations: A Bridge between Equilibrium and Off-Equilibrium. Journal of Statistical Physics. 1999. Vol. 97, no. 3-4. Pp. 459-488. DOI: https://doi.org/10.1023/A:1004602906332.

13. Mezard M., Parisi G. Thermodynamics of Glasses: a First Principles Computation. J. of Phys.: Condens. Matter. 1999. Vol. 11. Pp. A157-A165. DOI: https://doi.org/10.1088/0953-8984/11/10A/011.

14. Robles M., López de Haro M., Santos A., Bravo Yuste S. Is There a Glass Transition for Dense Hard-Sphere Systems? The Journal of Chemical Physics. 1998. Vol. 108, no. 3. Pp. 1290-1291. DOI: https://doi.org/10.1063/1.475499.

Статья поступила в редакцию 23.11.2020; одобрена после рецензирования 30.11.2020; принята к публикации 10.12.2020. 